\title{
A Particle on a Moving Plane with Coulomb Friction \\ O. Zubelevich
}

This paper is concerned with the motion of a particle on a horizontal vibrating plane with Coulomb friction. It is proved that, when some constant force is added, the system has a periodic solution.

Keywords: classical mechanics, systems with friction, Filippov's systems, periodic solutions, differential inclusions

\section{Formulation of the problem}

The purpose of this note is to draw the attention of specialists to some results of A.F. Filippov, which the author believes to be of crucial importance to gain insight into the dynamics of systems with Coulomb friction.

The theory of A.F. Filippov $[5,6]$ is considered using the following problem as an example.

A rough horizontal plane $\Pi$ undergoes translational motion in a prescribed manner without changing height, in such a way that each of its points executes periodic motion with period $\omega>0$.

A particle $P$ of mass $m$ slides on the plane in a standard gravitational field. The particle is acted upon by the force of Coulomb friction with coefficient $\sigma>0$ and by the gravity force, which is balanced by the normal reaction of the plane.

In addition, the particle is acted upon by constant force $\boldsymbol{G}$ directed along the plane $\Pi$.

The problem is: Can the constant force $\boldsymbol{G}$ be chosen so that $\omega$-periodic motions of the particle exist in this system?

Received June 30, 2019

Accepted August 10, 2019

This research was supported by a grant of the Russian Science Foundation (project No. 19-71-30012).

Oleg Zubelevich

ozubel@yandex.ru

Department of Theoretical Mechanics and Mechatronics,

Faculty of Mechanics and Mathematics, Lomonosov Moscow State University

Leninskiye Gory 1, GSP-1, Moscow, 119991 Russia

Steklov Mathematical Institute of the Russian Academy of Sciences

2-nd Krestovskii per. 12-179, Moscow, 129110 Russia 
A positive answer to this question is provided by Theorem 1, which is formulated and proved below.

The problem of the motion of a particle on a rough plane undergoing circular translational motion with constant frequency was investigated by N. E. Joukowski [4], see also [3].

The motion of a particle on an inclined plane, which undergoes periodic translational motion along a horizontal straight line, is considered in [1].

We now turn to formalizing the problem. It is convenient to choose dimensions of the physical quantities so that $\omega=1, g=1, m=1$.

We define two coordinate systems: a laboratory frame $O x y z$ with the plane $O x y$ coinciding with the plane $\Pi$, and a frame $A \xi \eta \zeta$ attached to the plane,

$$
A \in \Pi, \quad e_{x}=e_{\xi}, \quad e_{y}=e_{\eta}, \quad e_{z}=e_{\zeta} .
$$

Accordingly, the law of motion of any point $B$ of the plane $\Pi$ is described by the vector function

$$
\boldsymbol{O B}(t)=\boldsymbol{O A}(t)+\boldsymbol{A B} .
$$

The vector function $\boldsymbol{O} \boldsymbol{A}(t) \in C^{2}[0, \infty)$ is assumed to be given. According to the condition, it has period 1; the vector $\boldsymbol{A} \boldsymbol{B}$ does not depend on time.

The law of motion of the particle $P$ is defined by the radius vector $\boldsymbol{O P}(t)=\boldsymbol{O A}(t)+\boldsymbol{A P}(t)$.

Accordingly,

$$
\boldsymbol{v}=\frac{d}{d t} \boldsymbol{O P}(t)
$$

is the absolute velocity of the particle;

$$
\boldsymbol{u}(t)=\frac{d}{d t} \boldsymbol{A P}(t)=\boldsymbol{v}(t)-\boldsymbol{V}(t)
$$

is the velocity of the particle relative to the plane,

$$
\boldsymbol{V}(t)=\frac{d}{d t} \boldsymbol{O} \boldsymbol{A}(t)
$$

is the velocity of any point of the plane in the laboratory frame.

We note that, since the function $\boldsymbol{V}$ is a derivative of the periodic function, it is on average equal to zero; the same holds true for its derivative $\dot{\boldsymbol{V}}$.

Let us write the equations of motion:

$$
\dot{\boldsymbol{u}}=\boldsymbol{F}(t, \boldsymbol{u})+\boldsymbol{G}, \quad \boldsymbol{F}(t, \boldsymbol{u})=-\sigma \frac{\boldsymbol{u}}{|\boldsymbol{u}|}-\dot{\boldsymbol{V}}, \quad \boldsymbol{u} \neq 0
$$

Let $|\cdot|$ denote the standard Euclidean norm. The constant vector $\boldsymbol{G}$ is to be defined.

Since all vectors lie in the plane $\Pi$, we will assume in what follows that $\boldsymbol{u} \in \mathbb{R}^{2}$ can be decomposed by basis vectors $\boldsymbol{e}_{x}, \boldsymbol{e}_{y}$.

The right-hand side of (1.1) cannot be extended to the straight line $\{t \in \mathbb{R}, \boldsymbol{u}=0\}$ continuously. Thus, a standard definition of the solution makes no sense. We will search for periodic solutions in the class of generalized solutions in the sense of Filippov [5]. 


\subsection{Generalized solutions in the sense of Filippov}

As above, the vectors of our physical Euclidean space are denoted in bold face. The vectors of abstract $m$-dimensional space are denoted in normal font: $x=\left(x^{1}, \ldots, x^{m}\right)^{T} \in \mathbb{R}^{m}$.

Next, we let conv $U$ denote a closed convex shell of the set $U \subset \mathbb{R}^{m}$. Recall that the intersection of all convex closed sets containing $U$ is called a closed convex shell.

We also let

$$
B_{s}(x)=\left\{y \in \mathbb{R}^{m}|| y-x \mid<s\right\}
$$

denote an open ball in $\mathbb{R}^{m}$, and $\mu$ the Lebesgue measure in $\mathbb{R}^{m}$.

Let the map $f:\left[t_{1}, t_{2}\right] \times \mathbb{R}^{m} \rightarrow \mathbb{R}^{m}$ be Lebesgue measurable.

Definition 1 ([5]). We call the absolutely continuous function $x(t) \in C\left(\left[t_{1}, t_{2}\right], \mathbb{R}^{m}\right)$ a generalized solution to the equation

$$
\dot{x}=f(t, x)
$$

if for almost all $t \in\left[t_{1}, t_{2}\right]$ the inclusion

$$
\dot{x}(t) \in \bigcap_{\delta>0} \bigcap_{\mu(N)=0} \operatorname{conv} f\left(t, B_{\delta}(x(t)) \backslash N\right)
$$

takes place. The intersection is taken for all measurable sets $N \subset \mathbb{R}^{m}$ of the Lebesgue zero measure: $\mu(N)=0$.

$f\left(t, B_{\delta}(x(t)) \backslash N\right) \subset \mathbb{R}^{m}$ denotes an image of the set $B_{\delta}(x(t)) \backslash N$ for the map $x \mapsto f(t, x)$ with fixed $t$.

If the vector field $f$ is continuous, then the set on the right-hand side of (1.3) consists of the only element $f(t, x(t))$, and Definition 1 becomes a standard definition of the solution of the differential equation.

By Theorem 2 [5], the property of the function can be a generalized solution and does not depend on the choice of a system of coordinates $x$.

\section{The main theorem}

As noted above, the function $\boldsymbol{F}$ cannot be extended to the axis $\{(t, 0)\}$ continuously. However, it does not matter from the viewpoint of Definition 1 how exactly we extend the function $\boldsymbol{F}$ to the straight line $\{(t, 0)\}$. Note that this definition gives exactly the result that we expect to obtain from the theory of Coulomb friction.

Indeed, the set

$$
W=\bigcap_{\delta>0} \bigcap_{\mu(N)=0} \operatorname{conv}\left(\boldsymbol{F}\left(t, B_{\delta}(0) \backslash N\right)+\boldsymbol{G}\right)
$$

does not depend on the values of $\boldsymbol{F}(t, 0)$, since one of the sets of zero measure $N$ necessarily contains the point $\boldsymbol{u}=0$, and the value of $\boldsymbol{F}(t, 0)$, no matter what we choose it to be, does not affect the set (2.1).

If velocity $\boldsymbol{u}(t)$ is not zero on the time interval $\left(t^{\prime}, t^{\prime \prime}\right)$, then $\boldsymbol{u}(t)$ is a smooth function of time that satisfies Eq. (1.1) in the usual sense.

If the particle is at rest relative to the plane $\Pi$ on the interval $t \in\left(\tau^{\prime}, \tau^{\prime \prime}\right)$, then, in accordance with the definition of a generalized solution, we have

$$
\dot{\boldsymbol{u}}(t)=0 \in W=\bar{B}_{\sigma}(-\dot{\boldsymbol{V}}(t)+\boldsymbol{G}), \quad t \in\left(\tau^{\prime}, \tau^{\prime \prime}\right) .
$$


The little line denotes the closure of the ball. Then it follows from (2.2) that

$$
|-\dot{\boldsymbol{V}}(t)+\boldsymbol{G}| \leqslant \sigma .
$$

This is in agreement with the definition of Coulomb friction.

REMARK 1. By Theorem 10 [5], the generalized solution of the system (1.1) is unique forward in time at any given initial condition of $\boldsymbol{u}\left(t_{0}\right)$.

Theorem 1. There exists a constant force $\boldsymbol{G}$ such that the system (1.1) has a 1-periodic generalized solution $\boldsymbol{u}(t) \cdot \int_{0}^{1} \boldsymbol{u}(t) d t=0$, and hence the law of motion $\boldsymbol{O P}(t)$ is also a 1-periodic function of class $C^{1}$.

REMARK 2. The solution obtained in Theorem 1 corresponds to motion where the relative velocity of the particle $P$ can vanish, the position of the particle can for some time remain unchanged relative to the plane $\Pi$, and then the particle can start moving again.

If $|\dot{\boldsymbol{V}}(t)|<\sigma$ for all $t$, then the periodic motion can be the state of rest of the particle relative to the plane $\Pi$. For motions with segments $\boldsymbol{u} \neq 0$ to exist, it is necessary that $|\dot{\boldsymbol{V}}(t)|>\sigma$ for some $t$.

In the next section we formulate two theorems that are necessary to prove Theorem 1. A proof of Theorem 1 is given in Section 4 .

\section{Two general theorems}

\subsection{Approximation theorem}

Let the map

$$
f:\left[t_{1}, t_{2}\right] \times \mathbb{R}^{m} \rightarrow \mathbb{R}^{m}, \quad k \in \mathbb{N}
$$

be Lebesgue measurable. For simplicity we impose a stronger condition than each of conditions "A" and "B" from [5], namely, we will assume that the map $f$ is bounded:

$$
\sup \left\{|f(t, x)| \mid(t, x) \in\left[t_{1}, t_{2}\right] \times \mathbb{R}^{m}\right\}<\infty .
$$

This condition is obviously satisfied for the right-hand side of (1.1).

Theorem 2 ([5]). Assume that there exists a sequence of functions $g_{k}, f_{k} \in C\left(\left[t_{1}, t_{2}\right] \times\right.$ $\left.\mathbb{R}^{m}, \mathbb{R}^{m}\right)$ such that for almost all $(t, x)$ the inclusion

$$
f_{k}(t, x) \in \bigcap_{\mu(N)=0} \operatorname{conv} f\left(t, B_{\delta_{k}}(x) \backslash N\right), \quad \delta_{k} \rightarrow 0, \quad k \in \mathbb{N},
$$

is satisfied and

$$
\left\|\sup _{x \in \mathbb{R}^{m}} g_{k}(\cdot, x)\right\|_{L^{1}\left[t_{1} \cdot t_{2}\right]} \rightarrow 0, \quad k \rightarrow \infty .
$$

Then, if the sequence of solutions $x_{k}(t) \in C^{1}\left(\left[t_{1}, t_{2}\right], \mathbb{R}^{m}\right)$ to the systems

$$
\dot{x}_{k}=f_{k}\left(t, x_{k}\right)+g_{k}\left(t, x_{k}\right)
$$

is uniformly bounded:

$$
\sup _{k}\left\|x_{k}\right\|_{C\left[t_{1}, t_{2}\right]}<\infty
$$

then it contains a subsequence $\left\{x_{k_{j}}\right\}$ uniformly converging on $\left[t_{1}, t_{2}\right]$, and the limit of this subsequence is a generalized solution to the system (1.2). 


\subsection{Existence theorem}

Consider a system of differential equations in $\mathbb{R}^{m}=\left\{x=\left(x^{1}, \ldots, x^{m}\right)\right\}$ with time-periodic right-hand side

$$
\dot{x}=p(t, x)+G, \quad p(t+1, x)=p(t, x) .
$$

The components of the vector $p$ are functions of class $C\left([0, \infty) \times \mathbb{R}^{m}, \mathbb{R}^{m}\right)$. The constant vector $G \in \mathbb{R}^{m}$ is the parameter of the problem.

Theorem 3. Assume that there exist positive constants $C_{1}, C_{2}, \gamma$ such that for all admissible $(t, x)$ the estimate

$$
|p(t, x)| \leqslant C_{1}+C_{2}|x|^{\gamma}
$$

is satisfied and

$$
\gamma<1
$$

Then one can find a constant vector $G$ such that the system (3.2) has a 1-periodic solution $x(t) \in C^{1}\left([0, \infty), \mathbb{R}^{m}\right)$, and

$$
\int_{0}^{1} x(t) d t=0
$$

Moreover,

$$
|G| \leqslant C_{1}+C_{2} R^{\gamma}, \quad\|x\|_{C[0,1]} \leqslant R,
$$

where $R$ is some number satisfying the inequality

$$
4\left(C_{1}+C_{2} R^{\gamma}\right) \leqslant R
$$

Remark 3. The number $R>0$, which satisfies inequality (3.5), exists by virtue of condition (3.4).

Proof. Denote

$$
\langle u(\cdot)\rangle=\int_{0}^{1} u(t) d t, \quad u \in C[0,1]
$$

Introduce a Banach space

$$
E=\left\{y(t) \in C\left([0,1], \mathbb{R}^{m}\right) \mid y(0)=y(1), \quad\langle y\rangle=0\right\}
$$

with the norm $\|y\|=\max _{t \in[0,1]}|y(t)|$. Define the continuous map $\mathcal{F}: E \rightarrow E$ by the formula

$$
\mathcal{F}(y)=\int_{0}^{t}(p(s, y(s))-\langle p(\cdot, y(\cdot))\rangle) d s-\left\langle\int_{0}(p(s, y(s))-\langle p(\cdot, y(\cdot))\rangle) d s\right\rangle .
$$

By virtue of inequality (3.3) we have

$$
\|\mathcal{F}(y)\| \leqslant 4\left(C_{1}+C_{2}\|y\|^{\gamma}\right) .
$$

This implies that the map $\mathcal{F}$ translates the ball

$$
B_{R}=\{y \in E \mid\|y\| \leqslant R\}
$$

into itself: $\mathcal{F}\left(B_{R}\right) \subseteq B_{R}$.

Moreover, the image $\mathcal{F}\left(B_{R}\right)$ is an equicontinuous set. 
Indeed, denoting $u(t)=\mathcal{F}(y), \quad y \in B_{R}$, we obtain

$$
\left|u\left(t^{\prime \prime}\right)-u\left(t^{\prime}\right)\right| \leqslant 2\left(C_{1}+C_{2} R^{\gamma}\right)\left|t^{\prime \prime}-t^{\prime}\right|, \quad t^{\prime}, t^{\prime \prime} \in[0,1] .
$$

Consequently, by the Ascoli theorem [7], the image $\mathcal{F}\left(B_{R}\right)$ is relatively compact.

By the Schauder theorem of a fixed point [2], the map $\mathcal{F}$ has a fixed point $\tilde{y} \in B_{R}$ :

$$
\tilde{y}(t)=\int_{0}^{t}(p(s, \tilde{y}(s))-\langle p(\cdot, \tilde{y}(\cdot))\rangle) d s-\left\langle\int_{0}(p(s, \tilde{y}(s))-\langle p(\cdot, \tilde{y}(\cdot))\rangle) d s\right\rangle .
$$

According to the definition of space $E$, the function $\tilde{y}$ continues to the 1-periodic function $x(t) \in C[0, \infty)$. It is easy to check that Eq. (3.6) is satisfied for $x(t)$ at all $t \geqslant 0$ :

$$
x(t)=\int_{0}^{t}(p(s, x(s))-\langle p(\cdot, x(\cdot))\rangle) d s-\left\langle\int_{0}(p(s, x(s))-\langle p(\cdot, x(\cdot))\rangle) d s\right\rangle .
$$

Differentiating this equation with respect to $t$, we find

$$
\dot{x}(t)=p(t, x(t))-\langle p(\cdot, x(\cdot))\rangle .
$$

To complete the proof, it remains to set $G=-\langle p(\cdot, x(\cdot))\rangle$.

This proves Theorem 3 .

\section{Proof of Theorem 1}

Set $\boldsymbol{w}=\left(w^{1}, w^{2}\right) \in \mathbb{R}^{2}$. Let $\psi(\boldsymbol{w}) \in C^{\infty}\left(\mathbb{R}^{2}\right)$ be a nonnegative function such that

$$
\operatorname{supp} \psi \subset B_{1}(0), \quad \int_{\mathbb{R}^{2}} \psi(\boldsymbol{u}) d w^{1} d w^{2}=1 .
$$

Construct for this function a $\delta$-like sequence

$$
\psi_{k}(\boldsymbol{w})=k^{2} \psi(k \boldsymbol{w}), \quad k \in \mathbb{N} .
$$

Consider a family of smooth systems

$$
\dot{\boldsymbol{u}}=\boldsymbol{F}_{k}(t, \boldsymbol{u})+\boldsymbol{G}_{k}, \quad \boldsymbol{F}_{k}(t, \boldsymbol{u})=\int_{\mathbb{R}^{2}} \boldsymbol{F}(t, \boldsymbol{w}) \psi_{k}(\boldsymbol{w}-\boldsymbol{u}) d w^{1} d w^{2} .
$$

The vectors $\boldsymbol{G}_{k}$ are to be defined.

The inequality

$$
\left|\boldsymbol{F}_{k}(t, \boldsymbol{u})\right| \leqslant \sigma+\|\dot{\boldsymbol{V}}\|_{C[0,1]}
$$

follows immediately from (4.1) and (1.1). Thus, the right-hand side of the system (4.1) satisfies the conditions of Theorem 3 with constants, say,

$$
C_{1}=\sigma+\|\dot{\boldsymbol{V}}\|_{C[0,1]}, \quad C_{2}=\gamma=\frac{1}{2} .
$$


Indeed, from (4.2) we have

$$
\left|\boldsymbol{F}_{k}(t, \boldsymbol{u})\right| \leqslant \sigma+\|\dot{\boldsymbol{V}}\|_{C[0,1]} \leqslant C_{1}+\frac{1}{2}|\boldsymbol{u}|^{\frac{1}{2}} .
$$

By Theorem 3, for each number $k$ we can choose a constant vector $\boldsymbol{G}_{k}$ so that the systems (4.1) have 1-periodic solutions

$$
\boldsymbol{u}_{k}(t), \quad \int_{0}^{1} \boldsymbol{u}_{k}(t) d t=0,
$$

and the set of functions $\left\{\boldsymbol{u}_{k}\right\}$ is uniformly bounded on $[0,1]$ (and hence on $\mathbb{R}$, by virtue of periodicity of the functions $\boldsymbol{u}_{k}$ ), and $\sup _{k}\left|\boldsymbol{G}_{k}\right|<\infty$. Consequently, by virtue of the system (4.1), the set $\left\{\boldsymbol{u}_{k}\right\}$ is equicontinuous on $[0,1]$.

Consequently, by the Ascoli theorem, we can single out the subsequence $\left\{\boldsymbol{u}_{k_{j}}(t)\right\}$, which converges uniformly to the function $\boldsymbol{u}(t), \int_{0}^{1} \boldsymbol{u}(t) d t=0$ on $[0,1]$, and the sequence $\boldsymbol{G}_{k_{j}}$ converges to some vector $\boldsymbol{G}$.

Approximate the system (1.1) by

$$
\dot{\boldsymbol{u}}=\boldsymbol{F}_{k_{j}}(t, \boldsymbol{u})+\boldsymbol{G}+\boldsymbol{g}_{k_{j}}, \quad \boldsymbol{g}_{k_{j}}=\boldsymbol{G}_{k_{j}}-\boldsymbol{G} \rightarrow 0 .
$$

The systems (4.3) obviously constitute a subsequence of the systems (4.1).

We note that

$$
\boldsymbol{F}_{k_{j}}(t, \boldsymbol{u})+\boldsymbol{G}=\int_{\mathbb{R}^{2}}(\boldsymbol{F}(t, \boldsymbol{w})+\boldsymbol{G}) \psi_{k_{j}}(\boldsymbol{w}-\boldsymbol{u}) d w^{1} d w^{2},
$$

therefore, from the properties of the integral

$$
\boldsymbol{F}_{k_{j}}(t, \boldsymbol{u})+\boldsymbol{G} \in \bigcap_{\mu(N)=0} \operatorname{conv}\left(\boldsymbol{F}\left(t, B_{\delta_{j}}(\boldsymbol{u})\right)+\boldsymbol{G}\right), \quad \delta_{j}=\frac{1}{k_{j}} \rightarrow 0 .
$$

By Theorem 2, the function $\boldsymbol{u}(t)$ is the desired periodic solution.

This proves the theorem.

\section{References}

[1] Benedetti, A., Sornay, P., Dalloz, B., and Nicolas, M., Angular Particle Sliding Down a Transversally Vibrated Smooth Plane, Phys. Rev. E, 2012, vol. 85, no. 1, pt. 1, 011307, 8 pp.

[2] Agarwal, R., Meehan, M., and O'Regan, D., Fixed Point Theory and Applications, Cambridge Tracts in Math., vol. 141, Cambridge: Cambridge Univ. Press, 2001.

[3] Blekhman, I. I., Vibrational Mechanics: Nonlinear Dynamic Effects, General Approach, Applications, River Edge, N.J.: World Sci., 2000.

[4] Joukovsky, N. E., in Collected Papers: Vol. 8. Theory of Elasticity, Railways, Automobiles, A. P. Kotelnikov (Ed.), Moscow: Gostekhizdat, 1937, pp. 39-46 (Russian).

[5] Filippov, A.F., Differential Equations with Discontinuous Right-Hand Side, Mat. Sb. (N. S.), 1960, vol. 51(93), no. 1, pp. 99-128 (Russian).

[6] Filippov, A.F., Differential Equations with Discontinuous Righthand Sides, Math. Appl., vol.18, Dordrecht: Springer, 1988.

[7] Schwartz, L., Cours d'analyse: Vol. 2, Paris: Hermann, 1967. 